Pacific Journal of Mathematics

MODULAR PAIRS IN ORTHOMODULAR LATTICES 


\title{
MODULAR PAIRS IN ORTHOMODULAR LATTICES
}

\author{
ERIK A. SCHREINER
}

\begin{abstract}
Call an orthomodular lattice $L \quad M$-symmetric if $M(e, f)$ implies $M(f, e)$ for all $e, f \in L$ and $O$-symmetric if $M(e, f)$ implies $M\left(f^{\prime}, e^{\prime}\right)$. To check for these properties it is sufficient to consider only those modular pairs in which the two elements are complements. Every $O$-symmetric lattice is $M$-symmetric. In an atomic orthomodular lattice, $M$-symmetry is equivalent to the atomic exchange property.
\end{abstract}

The orthomodular lattice $L(H)$ of closed subspaces of a Hilbert space $H$ satisfies both symmetries but apparently for separate reasons. G. W. Mackey has shown [4, Theorem III-6] that two closed subspaces of $H$ form a dual modular pair in $L(H)$ if and only if their vector sum is a closed subspace. Thus the natural symmetry, $M$-symmetry, depends on the topology of $H . O$-symmetry arises in $L(H)$ as a consequence of properties of bounded linear operators on $H$ with closed ranges. It is the purpose of this paper to investigate these symmetries in arbitrary orthomodular lattices. Recent results of A. Ramsay [8] and M. D. MacLaren [7] have shown $M$-symmetry to be of importance in the study of locally finite dimension lattices. The Baer $*$-semigroup coordinatization theory for orthomodular lattices, developed by D. J. Foulis [2], enables us to adapt the idea of an operator with a closed range and conveniently and naturally introduce $O$-symmetry into arbitrary orthomodular lattices.

Confining our attention to orthomodular lattices, we first establish certain general properties of modular pairs. Using these results we develop characterizations of $M$-symmetric lattices and $O$-symmetric lattices in Theorem 7 and Theorem 8. That $O$-symmetry implies $M$-symmetry is established in Theorem 9 . In $\S 4$, atomic orthomodular lattices satisfying the atomic exchange property introduced by MacLane [5] are considered. Using specializations of the approach and procedure used by Ramsay in [8] it is shown that $M$-symmetry is equivalent to the atomic exchange property.

The author would like to express his appreciation to Professor D. J. Foulis for the many helpful suggestions he has made during the writing of this paper.

2. Modular pairs. In order to establish certain properties of modular pairs in orthomodular lattices, we shall make use of the Baer *-semigroup approach as defined and developed in $[1,2,3]$. We shall restrict our resume of definitions and results to a minimum of 
chiefly notational conventions. An orthomodular lattice is a lattice $L$ with 0 and 1 which is equipped with an orthocomplementation ': $L \rightarrow L$ which satisfies the orthomodular identity $e \leqq f \Rightarrow f=e \vee\left(f \wedge e^{\prime}\right)$. Recall that an orthocomplementation maps each element $e \in L$ onto a complement $e^{\prime}$ of $e$ in $L$ in such a way that $e^{\prime \prime}=e$ and $e \leqq f \Rightarrow f^{\prime} \leqq e^{\prime}$ for all $e, f \in L$. Examples and basic properties of orthomodular lattices may be found in $[1,2,3]$. While some of the following concepts make sense in arbitrary lattices we shall make the following restriction; $L$ will always represent an orthomodular lattice.

If $e, f \in L$, the ordered pair $(e, f)$ is called a modular pair, written $M(e, f)$, if $g \in L$ with $g \leqq f$ implies $g \vee(e \wedge f)=(g \vee e) \wedge f$. For $e, f \in L$, we say that e commutes with $f$, written $e C f$, if $e=(e \wedge f) \vee$ $\left(e \wedge f^{\prime}\right)$. If $e \leqq f$, the interval $L(e, f)=\{g \in L: e \leqq g \leqq f\}$ is an orthomodular lattice with orthocomplementation $g \rightarrow g^{\sharp}=e \vee\left(f \wedge g^{\prime}\right)=$ $\left(e \vee g^{\prime}\right) \wedge f$.

In a Baer *-semigroup $S, P^{\prime}=P^{\prime}(S)$ denotes the set of all closed projections, i.e., $e \in P^{\prime}$ if $e=e^{*}=e^{2}$ and $e=e^{\prime \prime}$. In $S$ one may generalize the concept of a bounded linear operator on a Hilbert space with closed range as follows: [1, p. 890] $x \in S$ is range-closed if $g \in P^{\prime}(S), g \leqq x^{\prime \prime}$ and $\left(g x^{*}\right)^{\prime \prime}=\left(x^{*}\right)^{\prime \prime}$ imply $g=x^{\prime \prime}$. This is equivalent to the condition $\left[\left(e x^{*}\right)^{\prime} x\right]^{\prime \prime}=e^{\prime} \wedge x^{\prime \prime}$ for all $e \in P^{\prime}$. We shall use the following result of Foulis [3, Theorem 28].

THeOREM 1. Let $S$ be a Baer *-semigroup and e, $f$ elements of the orthomodular lattice $P^{\prime}(S)$. Then $M(e, f)$ in $P^{\prime}(S)$ if and only if $e^{\prime} f$ is range closed in $S$. in $S$.

In $P^{\prime}(S)$ one has $e \wedge f=\left(e^{\prime} f\right)^{\prime} f$ and $e C f$ if and only it $e f=f e$

Lemma 2. Let $S$ be a Baer *-semigroup, $x, y \in S$. Then $x y$ range-closed implies $x^{\prime \prime} y$ is range-closed. Conversely, if $x$ and $x^{\prime \prime} y$ are range-closed, then $x y$ is range-closed.

Proof. Suppose $x y$ is range-closed and $g \in P^{\prime}(S)$ with $g \leqq\left(x^{\prime \prime} y\right)^{\prime \prime}$ and $\left(g y^{*} x^{\prime \prime}\right)^{\prime \prime}=\left(y^{*} x^{\prime \prime}\right)^{\prime \prime}$. Then $g \leqq(x y)^{\prime \prime}$ and $\left(g y^{*} x^{*}\right)^{\prime \prime}=\left(\left(g y^{*} x^{*}\right)^{\prime \prime} x^{*}\right)^{\prime \prime}=$ $\left(y^{*} x^{\prime \prime} x^{*}\right)^{\prime \prime}=\left(y^{*} x^{*}\right)^{\prime \prime}$. Hence $g=(x y)^{\prime \prime}=\left(x^{\prime \prime} y\right)^{\prime \prime}$ so $x^{\prime \prime} y$ is range-closed.

Conversely, let $x$ and $x^{\prime \prime} y$ be range-closed. Then

$$
\begin{aligned}
{\left[\left(e y^{*} x^{*}\right)^{\prime} x y\right]^{\prime \prime} } & =\left[\left\{\left(\left(e y^{*}\right)^{\prime \prime} x^{*}\right)^{\prime} x\right\}^{\prime \prime} y\right]^{\prime \prime}=\left[\left(\left(e y^{*}\right)^{\prime} \wedge x^{\prime \prime}\right) y\right]^{\prime \prime} \\
& =\left[\left(e y^{*} x^{\prime \prime}\right)^{\prime} x^{\prime \prime} y\right]^{\prime \prime}=e^{\prime} \wedge(x y)^{\prime \prime}
\end{aligned}
$$

for all $e \in P^{\prime}(S)$ so $x y$ is range-closed.

Applying Theorem 1 and Lemma 2 one has for $e \in P^{\prime}(S)$ and a range-closed element $x \in S$ that $x e$ is range-closed if and only if $M\left(x^{\prime}, e\right)$. 
Therefore, if $x$ is range-closed and $x e=e x$, xe must be range-closed, for $x e=e x$ implies that $x^{\prime} e=e x^{\prime}$, so $x^{\prime} C e$ and hence $M\left(x^{\prime}, e\right)$.

THeorem 3. Let $e, f, g \in L=P^{\prime}(S)$. If $M(e, f), g C e$ and $g C f$, then:
(i) $M(e \vee g, f \vee g)$.
(ii) $M(e \wedge g, f \wedge g)$.
(iii) $M(e, f \wedge g)$.
(iv) $M(e \vee g, f)$.

Proof. By Theorem 1, $e^{\prime} f$ is range-closed. The hypothesis on $g$ implies that $g$ and $g^{\prime}$ permute with $e^{\prime}$ and $f$ and thus with $e^{\prime} f$. Now $(e \vee g)^{\prime}(f \vee g)=e^{\prime} g^{\prime}\left(f^{\prime} g^{\prime}\right)^{\prime}=e^{\prime}\left(f \wedge g^{\prime}\right)=e^{\prime} f g^{\prime}$ which is range-closed by the preceeding remark. Thus $M(e \vee g, f \vee g)$. The other three cases may be proved in a similar manner or by direct lattice theoretical arguments.

Of the four other possible alterations of the pair $(e, f)$ using $g$, the pairs $(e \vee g, f \wedge g)$ and $(e \wedge g, f \wedge g)$ are automatically modular since the elements are comparable. The pairs $(e, f \vee g)$ and $(e \wedge g, f)$ are not necessarily modular.

One may derive another result as a consequence of Lemma 2 which amounts to an essential simplification of part of a theorem due to Foulis [1, Theorem 10].

THEOREM 4. The orthomodular lattice $L=P^{\prime}(S)$ is modular if and only if $S_{0}$, the sub-Baer *-semigroup generated by the closed projections in $S$ is range-closed.

Proof. We point out that for an arbitrary orthomodular lattice $L$ there are, unfortunately, many Baer *-semigroups that coordinatize $L$. However, there is a unique (up to isomorphism) minimum one, namely $S_{0}$, coordinatizing $L$. If $S_{0}$ is range-closed, that is, if every element of $S_{0}$ is range-closed, then for all $e, f \in L, e^{\prime} f$ is range-closed so $M(e, f)$ and $L$ is modular. Conversely, suppose $L$ is modular. We use induction on the number of closed projection factors in the element $x \in S_{0}$. If $x=e \in P^{\prime}(S)$ then $x$ is range-closed. Now assume that all products of $n$ closed projections are range-closed and let $x=$ $e_{1} e_{2} \cdots e_{n} e_{n+1}, e_{i} \in P^{\prime}(S)$ Since $e_{1} e_{2} \cdots e_{n}$ is range-closed and $M\left(\left(e_{1} e_{2} \cdots e_{n}\right)^{\prime}\right.$, $\left.e_{n+1}\right)$, by the remark following Lemma $2, x$ is range-closed

The pair $(e, f)$ is not modular if there is a $g<f$ with $g \vee(e \wedge f)<(g \vee e) \wedge f$. Letting $h=g \vee(e \wedge f)$ one has $h \vee$ $(e \wedge f)<(h \vee e) \wedge f$ where $e \wedge f<h<f$. As a result, the pair $(e, f)$ 
is modular in $L$ if and only if it is modular in $L(e \wedge f, e \vee f)$. More generally, if $e, f \in L(a, b)$, then $(e, f)$ is modular in $L$ if and only if $(e, f)$ is modular in $L(a, b)$. This observation simplifies our considerations.

The ordered pair $(e, f)$ is said to be a dual modular pair, written $M^{*}(e, f)$, if $h \geqq f$ implies $h \wedge(e \vee f)=(h \wedge e) \vee f$. As the final result of this section, we collect a number of facts about modular and dual modular pairs.

THeOREM 5. Let $e, f \in L$. Then:

(i) $M^{*}(e, f)$ if and only if $M\left(e^{\prime}, f^{\prime}\right)$.

(ii) If $e, f \in L(a, b), M^{*}(e, f)$ in $L$ if and only if $M^{*}(e, f)$ in $L(a, b)$.

(iii) $M(e, f)$ for all $f \in L$ if and only if $M^{*}(e, f)$ for all $f \in L$.

(iv) $M(e, f)$ for all $f \in L$ if and only if $M\left(e^{\prime}, f\right)$ for all $f \in L$.

(v) $M(e, f)$ for all $e \in L$ if and only if $M(c, f)$ for all complements $c$ of $f$ in $L$.

(vi) If $e \wedge f=0, e_{1} \leqq e, f_{1} \leqq f$, then $M(e, f)$ implies $M\left(e_{1}, f_{1}\right)$.

3. Symmetries of modular pairs. In this section we consider some general properties of two symmetry conditions on modular pairs.

Definition. An orthomodular lattice $L$ is said to be $M$-symmetric if for all $e, f \in L, M(e, f)$ implies $M(f, e)$.

An $M$-symmetric lattice is sometimes said to be semi-modular. Referring to the remark following Theorem 3 , if $L$ is $M$-symmetric, we do have $M(e, f), g C e$ and $g C f$ imply $M(e, f \vee g)$ and $M(e \wedge g, f)$. However, these implications do not imply that $L$ is $M$-symmetric.

If $P$ is any lattice property, then a lattice is relatively $P$ if every interval $L(a, b)$ satisfies $P$. Since we have for $e, f \in L(a, b)$ that $M(e, f)$ in $L$ if and only if $M(e, f)$ in $L(a, b)$, it follows that an $M$-symmetric lattice is relatively $M$-symmetric.

The second type of symmetry to be considered considered concerns the ordered pairs $(e, f)$ and $\left(f^{\prime}, e^{\prime}\right)$. In terms of coordinatizing Baer *-semigroups this arises in the following natural manner. In the semigroup of all bounded operators on a Hilbert space, it is known that an operator is range-closed if and only if its adjoint is rangeclosed. Thus if $e^{\prime}$ and $f$ are projections, then $e^{\prime} f$ is range-closed if and only if $\left(e^{\prime} f\right)^{*}=f e^{\prime}$ is range-closed. Using Theorem 1, this translates into $M(e, f)$ if and only if $M\left(f^{\prime}, e^{\prime}\right)$. The orthocomplementation involved is reflected in the following terminology.

Definition. An orthomodular lattice $L$ is said to be $O$-symmetric 
if for all $e, f \in L, M(e, f)$ implies $M\left(f^{\prime}, e^{\prime}\right)$.

The third possible symmetry, connecting $(e, f)$ and $\left(e^{\prime}, f^{\prime}\right)$, will not be considered since there seems to be no natural way in which it occurs. Morever, it is a composite of $M$-symmetry and $O$-symmetry.

Now, using the fact that the relative orthocomplement of $g \in L(a, b)$ is $g^{\sharp}=\left(g^{\prime} \wedge b\right) \vee a$ and Theorem 3, we derive the following result.

THEOREM 6. If $e, f \in L(a, b)$ then $M\left(f^{\sharp}, e^{\sharp}\right)$ if and only if $M\left(f^{\prime}, e^{\prime}\right)$. Thus an O-symmetric lattice is relatively O-symmetric.

Proof. Assume $M\left(f^{\sharp}, e^{\sharp}\right)$. Since $f^{\sharp}$ and $e^{\sharp}$ both commute with $a^{\prime}, M\left(f^{\sharp}, e^{\sharp}\right)$ implies $M\left(f^{\sharp} \wedge a^{\prime}, e^{\sharp} \wedge a^{\prime}\right)$. Since these two elements commute with $b^{\prime}$ we obtain $M\left(\left(f^{*} \wedge a^{\prime}\right) \vee b^{\prime},\left(e^{\sharp} \wedge a^{\prime}\right) \vee b^{\prime}\right)$. But $\left(f^{\#} \wedge a^{\prime}\right) \vee b^{\prime}=\left(\left[\left(f^{\prime} \wedge b\right) \vee a\right] \wedge a^{\prime}\right) \vee b^{\prime}=f^{\prime}$ and $\left(e^{\#} \wedge a^{\prime}\right) \vee b^{\prime}=e^{\prime}$ so we have $M\left(f^{\prime}, e^{\prime}\right)$. Conversely, since $f^{\prime}$ and $e^{\prime}$ commute with $b$ and since $f^{\prime} \wedge b$ and $e^{\prime} \wedge b$ commute with $a, M\left(f^{\prime}, e^{\prime}\right)$ implies $M\left(\left(f^{\prime} \wedge b\right) \vee a\right.$, $\left(e^{\prime} \wedge\right.$ b) $\left.\vee a\right)$, that is, $M\left(f^{*}, e\right)^{\sharp}$. The second conclusion now follows immediately.

The mapping $g \rightarrow g \wedge a^{\prime}$ is an isomorphism from $L(a, b)$ onto $L\left(0, b \wedge a^{\prime}\right)$ that preserves relative orthocomplements. Denoting this mapping by $\varphi$, we have for $e, f \in L(a, b), M(e, f)$ in $L(a, b)$ if and only if $M(e,, f \varphi)$ in $L\left(0, b \wedge a^{\prime}\right)$. Since the first condition is equivalent to $M(e, f)$ in $L$ we see that to investigate $L$ for $M$-symmetry it is enough to consider pairs $(e, f)$ such that $e \wedge f=0$. This establishes the equivalence of (i) and (ii) in the following characterization.

THEOREM 7. Let $L$ be an orthomodular lattice. The following conditions are mutually equivalent:

(i) $L$ is $M$-symmetric.

(ii) For $e, f \in L$ with $e \wedge f=0, M(e, f)$ implies $M(f, e)$.

(iii) For $e, f \in L$ with e a complement of $f, M(e, f)$ implies $M(f, e)$.

Proof. We need only establish that (iii) $\Rightarrow$ (ii). Thus suppose that (iii) holds. Let $e \wedge f=0$ and $M(e, f)$. Then $e \vee(e \vee f)^{\prime}$ is a complement of $f$ and since $e$ and $f$ commute with $(e \vee f)^{\prime}, M(e, f)$ implies $M\left(e \vee(e \vee f)^{\prime}, f\right)$. By (iii) we obtain $M\left(f, e \vee(e \vee f)^{\prime}\right)$. Applying Theorem 5 (vi) we have $M(f, e)$.

For $O$-symmetry we have a similar result.

THEOREM 8. Let $L$ be an orthomodular lattice. The following conditions are mutually equivalent:

(i) $L$ is O-symmetric.

(ii) For $e, f \in L$ with $e \wedge f=0, M(e, f)$ implies $M\left(f^{\prime}, e^{\prime}\right)$. 
(iii) For $e, f \in L$ with $e$ a complement of $f, M(e, f)$ implies $M\left(f^{\prime}, e^{\prime}\right)$.

Proof. (i) $\Leftrightarrow$ (ii). By Theorem 6, when considering the pair $(e, f)$ it is sufficient to consider the interval $L(e \wedge f, e \vee f)$. By the remark preceeding Theorem 7, it is enough to consider only intervals of the type $L(0, g)$.

(iii) $\Rightarrow$ (ii). Suppose $M(e, f)$ where $e \wedge f=0$. As $e$ and $f$ both commute with $(e \vee f)^{\prime}, M\left(e \vee(e \vee f)^{\prime}, f\right)$ by Theorem 3. Since $e \vee(e \vee f)^{\prime}$ is a complement of $f$, we have $M\left(f^{\prime}, e^{\prime} \wedge(e \vee f)\right)$. Now $f^{\prime}$ and $e^{\prime} \wedge(e \vee f)$ both commute with $(e \vee f)^{\prime}$ so, again by Theorem 3, we obtain $M\left(f^{\prime} \vee(e \vee f)^{\prime},\left[e^{\prime} \wedge(e \vee f)\right] \vee(e \vee f)^{\prime}\right)$ which is precisely $M\left(f^{\prime}, e^{\prime}\right)$.

That (ii) $\Rightarrow$ (iii) is clear.

We are now in a position to establish the following connection between the two symmetries.

Theorem 9. Every O-symmetric lattice is M-symmetric.

Proof. ${ }^{1}$ Let $L$ by $O$-symmetric, $e, f \in L$ with $e \wedge f=0$. By Theorem $5, M(e, f)$ implies $M(g, f)$ for all $g \leqq e$. Thus, for all $g \leqq e$, $M\left(f^{\prime}, g^{\prime}\right)$ which is equivalent to $M^{*}(f, g)$. This last statement implies $M(f, e)$.

Whether the converse of this theorem holds is still an open question. In addition to the similarities shown in Theorems 7 and 8 , both properties are preserved under the taking of direct products.

4. Atomic orthomodular lattices. For $a, b \in L$, if $a<b$ we say that $b$ covers $a$ if $a<c \leqq b$ implies $c=b$. An element that covers 0 is called an atom. $L$ is called atomic if every nonzero element dominates an atom. In an atomic orthomodular lattice every nonzero element is the supremum of the atoms it dominates. An atomic orthomodular lattice is said to satisfy the atomic exchange property (AEP) if $a$ and $b$ atoms with $a \leqq b \vee e$ and $a \geqq e$ implies $b \leqq a \vee e$.

Prime tools in the coordinatization theory of orthomodular lattices are the mappings $\varphi_{e}: L \rightarrow L$ defined for each $e \in L$ by $a \varphi_{e}=\left(\alpha \vee e^{\prime}\right) \wedge e$. $\varphi_{e}$ is called the Sasaki projection determined by $e$. Such mappings are monotone and preserve arbitrary suprema [2]. An atomic lattice is said to satisfy the atomic projection property (APP) if for all atoms $a$ and for all $e \in L, a \geqq e^{\prime}$ implies $a \varphi_{e}$ is an atom.

\footnotetext{
1 The author is indebted to the referee for this simple proof.
} 
Lemma 10. Let $L$ be an atomic orthomodular lattice. The following conditions are mutually equivalent:

(i) $a$ an atom, $a \wedge b=0$ implies $a \vee b$ covers $b$.

(ii) $L$ satisfies APP.

(iii) L satisfies AEP.

(iv) $b$ an atom, $x \leqq b \vee e$ and $x \not e$ imply $b \leqq x \vee e$.

(v) For all atoms $a \in L, M(a, e)$ for all $e \in L$.

The proof, which is routine, is omitted. If $L$ satisfies AEP it also satisfies the condition $a$ and $b$ cover $a \wedge b$ if and only if $a \vee b$ covers $a$ and $b$. In the sequel we make use of all these conditions.

LEMMA 11. Every $M$-symmetric, and hence every O-symmetric atomic lattice $L$ satisfies AEP.

Proof. For all atoms $a \in L$ and all $e \in L M(e, a)$ and hence $M(\alpha, e)$.

A nonempty subset $A$ of $L$ is an ideal if $a, b \in A$ implies $a \vee b \in A$ and $\boldsymbol{a} \in A, e \leqq a$ implies $e \in A$. If $A$ and $B$ are ideals, then $A \vee B=$ $\{e: e \leqq a \vee b$ for some $a \in A, b \in B\}$ is the smallest ideal containing $A$ and $B$. For $a, b \in L$, say that $a$ and $b$ are orthogonal, written $a \perp b$ if $a \leqq b^{\prime}$. For orthogonal elements write $a \oplus b$ for $a \vee b$. If $\left\{a_{i}: i=1,2, \cdots, n\right\}$ is an orthogonal family, that is, if $i \neq j$ implies $a_{i} \perp a_{j}$ we write $a_{1} \oplus a_{2} \oplus \cdots \oplus a_{n}$ for $a_{1} \vee a_{2} \vee \cdots \vee a_{n}$.

Henceforth, $L$ will always denote an atomic orthomodular lattice. We shall call $b \in L$ finite if $b=0$ or $b$ is the join of a finite number of atoms. Let $J$ denote the set of all finite elements in $L$. Minor modifications of the proof of [6, Theorem 4.1] enable us to show that if $L$ satisfies AEP then $J$ is an ideal of $L$. Moreover, if we define on $J$ the integer valued function $\operatorname{deg}(b)=\min \{n: b$ is the join of $n$ atoms $\}$ then for $b \in J, \operatorname{deg}(b)=n$ if and only if $b=a_{1} \oplus a_{2} \oplus \cdots \oplus a_{n}$ where each $a_{i}$ is an atom.

Lemma 12. If $L$ satisfies $\mathrm{AEP}$ and $b \in J$ then $b \varphi_{e} \in J$ for all $e \in L . \quad$ Moreover, $\operatorname{deg}\left(b \varphi_{e}\right) \leqq \operatorname{deg}(b)$. If $a \in L$ has a common complement with $b$ then $a \in J$ and $\operatorname{deg}(a)=\operatorname{deg}(b)$.

Proof. Let $b=a_{1} \oplus \cdots \oplus a_{n}, a_{i}$ atoms. Then $b \varphi_{e}=a_{1} \varphi_{e} \vee \cdots \vee$ $a_{n} \varphi_{e}$ and each nonzero $a_{i} \varphi_{e}$ is an atom by APP. Thus deg $\left(b \varphi_{e}\right) \leqq \operatorname{deg}(b)$. Now if $e$ is a complement of both $a$ and $b$, then $b \varphi_{e^{\prime}}=e^{\prime}$ so $e^{\prime} \in J$ with $\operatorname{deg}\left(e^{\prime}\right) \leqq \operatorname{deg}(b)$. Since $e^{\prime} \varphi_{b}=b, \operatorname{deg}(b)=\operatorname{deg}\left(e^{\prime}\right)$. Since $e^{\prime} \varphi_{a}=a$ and $a \varphi_{e^{\prime}}=e^{\prime}, a \in J$ and $\operatorname{deg}(a)=\operatorname{deg}\left(e^{\prime}\right)=\operatorname{deg}(b)$.

THeOREm 13. Let $L$ satisfy AEP. Then $b \in J$ implies $M(c, b)$ 
and $M(b, c)$ for all $c \in L$. Therefore $J$ is a modular sublattice of $L$.

Proof. Since the modularity of the pair $(b, c)$ depends only on the interval $L(b \wedge c, b \vee c)$ we may assume $b \wedge c=0$. Let $\operatorname{deg}(b)=n$ so $b=a_{1} \oplus \cdots \oplus a_{n}, a_{i}$ atoms. Let $e \leqq c$. To show $M(b, c)$ we must prove $h=(e \vee b) \wedge c \wedge e^{\prime}=0$. Assume $h \neq 0$. Since $h \leqq e, h \leqq a_{1} \vee e$ implies $a_{1} \leqq h \vee e \leqq c$ contradicting $b \wedge c=0$. Thus $h \leqq a_{1} \vee e$. If $h \leqq a_{1} \vee \cdots \vee a_{j-1} \vee e$, then $h \leqq a_{1} \vee \cdots \vee a_{j} \vee e$ implies $a_{j} \leqq$ $a_{1} \vee \cdots \vee a_{j-1} \vee h \vee e \leqq a_{1} \vee \cdots \vee a_{j-1} \vee c$. Let

$$
d=a_{1} \oplus \cdots \oplus a_{j-1} \oplus a_{j+1} \oplus \cdots \oplus a_{n}
$$

and join $d \vee c \vee(b \vee c)^{\prime}$ to both ends of the last inequality. This yields $1=d \vee c \vee(b \vee c)^{\prime}$. Since $d \wedge\left[c \vee(b \vee c)^{\prime}\right]=0, c \vee(b \vee c)^{\prime}$ is a common complement of $b$ and $d$. But then $n-1=\operatorname{deg}(d)=$ $\operatorname{deg}(b)=n$ by Lemma 12. This contradiction establishes that $h \geqq a_{1} \vee \cdots \vee a_{j} \vee e$. By induction, $h \neq 0$ implies $h \geqq b \vee e$. However, $h=(e \vee b) \wedge c \wedge e^{\prime}$ so we must have $h=0$. Thus $M(b, c)$ for all $c \in L$.

By Theorem 5 , to test $M(c, b)$ for all $c \in L$ it is sufficient to consider only complements of $b$. If $c$ is a complement of $b$ such that $(c, b)$ is not modular, there exists $e<b$ with $e<(e \vee c) \wedge b$. Since $(e \vee c) \wedge b \in J$ and $e$ have the common complement $c \vee(e \vee c)^{\prime}$, $\operatorname{deg}((e \vee c) \wedge b)=\operatorname{deg}(e)<\operatorname{deg}((e \vee c) \wedge b)$. This contradiction establishes the theorem.

Note that by Theorems 5 and 13 , if $b \in J$ we also have $M^{*}(c, b)$ for all $c \in L$.

A subset $A$ of $L$ is called join-dense in $L$ if every element of $L$ is the join of some subset of $A$. In an orthomodular lattices this is equivalent to the condition $b \neq 0$ implies $A \cap L(0, b)$ contains a nonzero element. We point out that completeness of $L$ is not necessary for this equivalence. If $L$ satisfies AEP, $J$ is a join-dense modular ideal of $L$. For nonzero $b \in L$, let $J(b)=J \cap L(0, b)$. If $L$ satisfies AEP, since $L(0, b)$ and $J$ are ideals of $L, J(b)$ is an ideal of $L$ as well as an ideal of $L(0, b)$.

We now generalize the theorem of Mackey mentioned in the introduction. Note that for $A, B \in L(H), A+B$ is closed and therefore equals $A \vee B$ if and only if there are no one-dimensional subspaces contained in $A \vee B$ but not in $A+B$. We first treat the analog of a finite dimensional subspace, in which case $A+B=A \vee B$ always holds.

LEMma 14. Let $L$ satisfy AEP. If $a \in L$ and $b \in J$, then $J(a \vee b)=J(a) \vee J(b)$. 


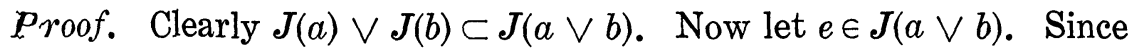
$e \vee b \in J,(e \vee b) \wedge a \in J(a)$ and $M(a, e \vee b)$ so $((e \vee b) \wedge a) \vee b=$ $e \vee b$. Thus $e \vee b \in J(a) \vee J(b)$ and since $e \leqq e \vee b, e \in J(a) \vee J(b)$.

THEOREM 15. Let $L$ be an atomic orthomodular lattice satisfying AEP. Then for $a, b \in L$ the following conditions are mutually equivalent:

(i) $J(a \vee b)=J(a) \vee J(b)$

(ii) $J(a) \vee J(b)$ is join-dense in $L(0, a \vee b)$.

(iii) $M^{*}(b, a)$.

Proof. That (i) $\Rightarrow$ (ii) is clear.

(ii) $\Rightarrow$ (iii). Let $a \leqq c \leqq a \vee b$. To show $c=a \vee(b \wedge c)$, it is enough to show $J(a) \vee J(b \wedge c)$ is join-dense in $L(0, c)$. Thus let $e \in L(0, c)$ with $e \neq 0$. Since $L(0, c) \subset L(0, a \vee b)$ there is an $f \in J(a)$ and $g \in J(b)$ with $(f \vee g) \wedge e \neq 0$. Set $e_{1}=(f \vee g) \wedge e$. By Theorem 13, $\boldsymbol{M}\left(g, f \vee e_{1}\right)$ so $f \vee\left(g \wedge\left(f \vee e_{1}\right)\right)=f \vee e_{1}$. Since $g \wedge\left(f \vee e_{1}\right) \in$ $L(0, b \wedge c), f \vee e_{1}$ and hence $e_{1}$ belongs to $J(a) \vee J(b \wedge c)$. Since $e_{1} \neq 0, J(a) \vee J(b \wedge c)$ is join-dense in $L(0, c)$. Thus $c=a \vee(b \wedge c)$. That is, $M^{*}(b, a)$.

(iii) $\Rightarrow$ (i). We need only show $J(a \vee b) \subset J(a) \vee J(b)$. Let $e \in J(a \vee b), g=e \varphi_{a^{\prime}}$ and $f=(e \vee a) \wedge a^{\prime} \varphi_{b}$. Since $M^{*}(b, a), f \vee g^{\prime}=1$. As $f \wedge g^{\prime}=0, g^{\prime}$ is a common complement of $g$ and $f$. Thus, by Lemma $12, e \in J$ implies $g \in J$ which in turn implies $f \in J$. Making use of the orthomodular identity and $M^{*}(b, a)$ we have $a \vee f=$ $a \vee(a \wedge b) \vee f=a \vee(b \wedge(a \vee e))=a \vee e$. Since $f \in J$, by Lemma $14, J(a \vee e)=J(a \vee f)=J(a) \vee J(f)$. Thus $f \leqq b$ implies

$$
e \in J(a \vee e) \subset J(a) \vee J(b)
$$

so (i) holds.

COROLLARY 16. An atomic orthomodular lattice is $M$-symmetric if and only if it satisfies AEP.

Proof. Necessity was established by Lemma 11. By Theorem 5, $M$-symmetry is equivalent to the symmetry of dual modular pairs. Sufficiency thus follows since condition (i) of Theorem 15 is symmetric in $a$ and $b$.

\section{REFERENCES}

1. D. J. Foulis, Conditions for the modularity of an orthomodular lattice, Pacific J. Math. 11 (1961), 889-895.

2. - A note on orthomodular lattices, Portugal. Math. 21, Fasc. 1 (1962), 65-72. 
3. __ Relative inverses in Baer *-semigroups, Mich. Math. J. 10 (1963), 65-84. 4. G. Mackey, On infinite-dimensional linear spaces, Trans. Amer. Math. Soc. 57 (1945), 155-207.

5. S. MacLane, A lattice formulation for transcendence degree and p-basis, Duke Math. J. 4 (1938), 455-468.

6. M. D. MacLaren, Atomic orthocomplemented lattices, Pacific J. Math. 14 (1964), 597-612.

7. - Nearly modular orthocomplemented lattices, Trans. Amer. Math. Soc. 114 (1965), 401-416

8. A. Ramsay, Dimension theory in complete orthocomplemented weakly modular lattices, Trans. Amer. Math. Soc. 116 (1965), 9-31.

Received August 9, 1965. The results in this paper form part of the author's doctoral dissertation (Wayne State University, 1964) written under the direction of Professor D. J. Foulis. The research was partially supported by a National Science Foundation Summer Fellowship.

WESTERN Michigan UNIVERSITY 


\section{PACIFIC JOURNAL OF MATHEMATICS}

\section{EDITORS}

H. SAMELSON

Stanford University

Stanford, California

J. P. JANS

University of Washington

Seattle, Washington 98105

\section{J. DuGUNDJI}

University of Southern California

Los Angeles, California 90007

RICHARD ARENS

University of California

Los Angeles, California 90024

\section{ASSOCIATE EDITORS}

E. F. BECKENBACH
B. H. NEUMANN

F. WOLF

K. YoSIDA

\section{SUPPORTING INSTITUTIONS}

\author{
UNIVERSITY OF BRITISH COLUMBIA \\ CALIFORNIA INSTITUTE OF TECHNOLOGY \\ UNIVERSITY OF CALIFORNIA \\ MONTANA STATE UNIVERSITY \\ UNIVERSITY OF NEVADA \\ NEW MEXICO STATE UNIVERSITY \\ OREGON STATE UNIVERSITY \\ UNIVERSITY OF OREGON \\ OSAKA UNIVERSITY \\ UNIVERSITY OF SOUTHERN CALIFORNIA
}

\author{
STANFORD UNIVERSITY \\ UNIVERSITY OF TOKYO \\ UNIVERSITY OF UTAH \\ WASHINGTON STATE UNIVERSITY \\ UNIVERSITY OF WASHINGTON \\ AMERICAN MATHEMATICAL SOCIETY \\ CHEVRON RESEARCH CORPORATION \\ TRW SYSTEMS \\ NAVAL ORDNANCE TEST STATION
}

Mathematical papers intended for publication in the Pacific Journal of Mathematics should be typewritten (double spaced). The first paragraph or two must be capable of being used separately as a synopsis of the entire paper. It should not contain references to the bibliography. Manuscripts may be sent to any one of the four editors. All other communications to the editors should be addressed to the managing editor, Richard Arens at the University of California, Los Angeles, California 90024 .

50 reprints per author of each article are furnished free of charge; additional copies may be obtained at cost in multiples of 50 .

The Pacific Journal of Mathematics is published monthly. Effective with Volume 16 the price per volume (3 numbers) is $\$ 8.00$; single issues, $\$ 3.00$. Special price for current issues to individual faculty members of supporting institutions and to individual members of the American Mathematical Society: $\$ 4.00$ per volume; single issues $\$ 1.50$. Back numbers are available.

Subscriptions, orders for back numbers, and changes of address should be sent to Pacific Journal of Mathematics, 103 Highland Boulevard, Berkeley 8, California.

Printed at Kokusai Bunken Insatsusha (International Academic Printing Co., Ltd.), No. 6, 2-chome, Fujimi-cho, Chiyoda-ku, Tokyo, Japan.

\section{PUBLISHED BY PACIFIC JOURNAL OF MATHEMATICS, A NON-PROFIT CORPORATION}

The Supporting Institutions listed above contribute to the cost of publication of this Journal, but they are not owners or publishers and have no responsibility for its content or policies. 


\section{Pacific Journal of Mathematics

Vol. 19, No. $3 \quad$ July, 1966

S. J. Bernau, The spectral theorem for unbounded normal operators .......

Lu-san Chen, Asymptotic behavior of solutions of parabolic equations of

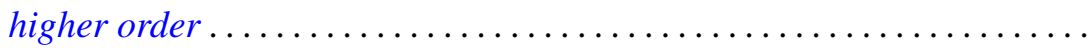

Lawrence William Conlon, An application of the Bott suspension map to the

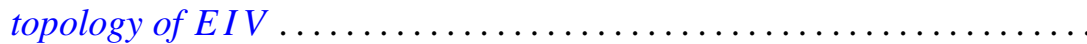

Neal Eugene Foland and John M. Marr, Sets with zero-dimensional kernels .........................................

Stanley Phillip Franklin and R. H. Sorgenfrey, Closed and image-closed

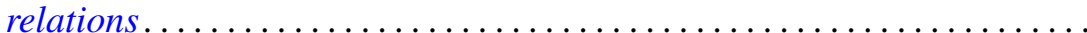

William Jesse Gray, A note on topological transformation groups with a

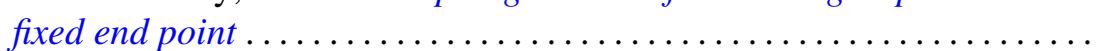

Myron Goldstein, $K$ - and L-kernels on an arbitrary Riemann surface ...... 449

George Joseph Kertz and Francis Regan, The exponential analogue of a generalized Weierstrass series .............................

Walter Leighton, On Liapunov functions with a single critical point ........ 467

Bernard Werner Levinger and Richard Steven Varga, On a problem of $O$.

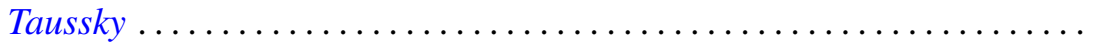

Lowell Duane Loveland, Tame subsets of spheres in $E^{3} \ldots \ldots \ldots \ldots \ldots . .489$

Erik Andrew Schreiner, Modular pairs in orthomodular lattices ......... 519

K. N. Srivastava, On dual series relations involving Laguerre polynomials ...............................

Arthur Steger, Diagonability of idempotent matrices.....

Walter Strauss, On continuity of functions with values in various Banach spaces...

Robert Vermes, On the zeros of a linear combination of polynomials ...

Elliot Carl Weinberg, On the scarcity of lattice-ordered matrix rings ....

Harold Widom, Toeplitz operators on $H_{p} \ldots \ldots \ldots \ldots \ldots$

Neal Zierler, On the lattice of closed subspaces of Hilbert space...

Irving Leonard Glicksberg, Correction to: "Maximal algebras and a theorem of Rado"

John Spurgeon Bradley, Correction to: "Adjoint quasi-differential operators of Euler type"

William Branham Jones, Erratum: "Duality and types of completeness in locally covex spaces".

Stanley P. Gudder, Erratum: "Uniqueness and existence properties of bounded observables" 